\title{
Overexpression of Salmonella enterica serovar Typhi recA gene confers fluoroquinolone resistance in Escherichia coli DH5 $\alpha$
}

\author{
M.A.M. Yassien and M.A. Elfaky \\ Department of Natural Products and Alternative Medicine/Microbiology, Faculty of Pharmacy, \\ King Abdulaziz University, Jeddah, Saudi Arabia
}

\begin{abstract}
A spontaneous fluoroquinolone-resistant mutant (STM1) was isolated from its parent Salmonella enterica serovar Typhi (S. Typhi) clinical isolate. Unlike its parent isolate, this mutant has selective resistance to fluoroquinolones without any change in its sensitivity to various other antibiotics. DNA gyrase assays revealed that the fluoroquinolone resistance phenotype of the STM1 mutant did not result from alteration of the fluoroquinolone sensitivity of the DNA gyrase isolated from it. To study the mechanism of fluoroquinolone resistance, a genomic library from the STM1 mutant was constructed in Escherichia coli DH5 $\alpha$ and two recombinant plasmids were obtained. Only one of these plasmids (STM1-A) conferred the selective fluoroquinolone resistance phenotype to $E$. coli $\mathrm{DH} 5 \alpha$. The chromosomal insert from STM1-A, digested with EcoRI and HindllI restriction endonucleases, produced two DNA fragments and these were cloned separately into pUC19 thereby generating two new plasmids, STM1-A1 and STM1-A2. Only STM1-A1 conferred the selective fluoroquinolone resistance phenotype to E. coli $\mathrm{DH} 5 \alpha$. Sequence and subcloning analyses of STM1-A1 showed the presence of an intact RecA open reading frame. Unlike that of the wild-type $E$. coli DH5 $\alpha$, protein analysis of a crude STM1-A1 extract showed overexpression of a $40 \mathrm{kDa}$ protein. Western blotting confirmed the $40 \mathrm{kDa}$ protein band to be RecA. When a RecA PCR product was cloned into pGEM-T and introduced into $E$. coli $\mathrm{DH} 5 \alpha$, the STM1-A11 subclone retained fluoroquinolone resistance. These results suggest that overexpression of RecA causes selective fluoroquinolone resistance in $E$. coli $\mathrm{DH} 5 \alpha$.
\end{abstract}

Key words: Salmonella enterica serovar Typhi; Fluoroquinolones; RecA

\section{Introduction}

Fluoroquinolones are synthetic antimicrobial agents with a broad spectrum of activity and potent antibacterial activity against Gram-negative bacteria (1). The extensive use of fluoroquinolones for the treatment of typhoid is one important factor that has led to the frequent isolation of fluoroquinolone-resistant isolates in developing countries in Africa and South Asia (2).

Fluoroquinolone resistance in Gram-negative bacteria involves mutations in DNA gyrase that confer various levels of resistance to fluoroquinolones or alterations in drug permeation across the bacterial cell membrane; such mutations can lead to fluoroquinolone resistance and resistance to unrelated classes of antimicrobial agent (3).

Fluoroquinolones are potent inducers of SOS responses in bacteria, and these responses result from induction of more than $\mathbf{2 0}$ genes or operons (4). The SOS response plays an important role in repairing the DNA damage caused by, for example, chemical treatments. The RecA protein, a ubiquitous bacterial recombination protein, plays a role in mediating SOS responses in bacteria. Through this response, the RecA protein may play a role in the development of fluoroquinolone resistance $(5,6)$.

A spontaneous fluoroquinolone-resistant mutant (STM1) was randomly isolated from a serovar Typhi clinical isolate of Salmonella enterica (S. Typhi). The results of DNA gyrase sensitivity testing of this mutant revealed that the development of fluoroquinolone resistance is not the result of mutation in DNA gyrase. The present study focused on identifying the mechanism of fluoroquinolone resistance in the STM1 mutant.

\section{Material and Methods}

\section{Bacteria and culture media}

The S. Typhi clinical isolate used herein and characterized by its sensitivity to fluoroquinolones was obtained from the Microbiology Laboratory in the King Abdulaziz

Correspondence: M.A.M. Yassien: <myassien61@yahoo.com>. 
University Hospital, Saudi Arabia. The fluoroquinoloneresistant mutant, STM1, was isolated randomly from the parental strain.

The bacterial strains and plasmids used in the present study are listed in Table 1. Unless otherwise noted, bacteria were grown in Luria-Bertani broth (LB, $10 \mathrm{~g}$ tryptone, $5 \mathrm{~g}$ yeast extract, $10 \mathrm{~g} \mathrm{NaCl} / \mathrm{L}$ ) under aerobic condition at $37^{\circ} \mathrm{C}$. Cell growth was monitored turbidimetrically at $600 \mathrm{~nm}$. All reagents were the purest available grade (Sigma Aldrich, USA), and the culture media were obtained from Oxoid (USA).

\section{Recombinant DNA techniques}

Unless otherwise indicated, standard protocols were used for isolation of plasmid and chromosomal DNA, bacterial transformation, and western blot analysis (7). Nucleotide sequences were determined by the dideoxy chain termination method (8) with a Terminator Cycle Sequencing kit (Applied Biosystems 3500 Genetic Analyzer, Applied Biosystems, USA). The Basic Local Alignment Search Tool (BLAST) program at the National Center for Biotechnology Information (www.ncbi.nlm.nih.gov/blst. cgi) was used to analyze the sequence data and assess the degree of DNA similarity between the sequences.

\section{Cloning the ciprofloxacin resistance gene}

Chromosomal DNA was prepared from S. Typhi STM1 cells by using an Ultra Clean DNA Isolation Kit (MO-BIO, USA). The DNA was partially digested with Sau3AI, and fragments of between 1 and $10 \mathrm{kbp}$ were separated by electrophoresis in $0.5 \%$ agarose gels and then purified by a Qiaquick Gel Extraction Kit (Qiagen, USA). The DNA fragments were ligated to pUC19 (digested with BamHI and dephosphorylated with bacterial alkaline phosphatase) with T4 DNA ligase (Boehringer Mannheim $\mathrm{GmbH}$., Germany). Competent Escherichia coli $\mathrm{DH} 5 \alpha$ cells, transformed with the recombinant plasmids, were spread on LB agar plates containing $0.5 \mu \mathrm{g} / \mathrm{mL}$ of ciprofloxacin and $100 \mu \mathrm{g} / \mathrm{mL}$ of ampicillin. The plates were incubated under aerobic conditions at $37^{\circ} \mathrm{C}$ for $24 \mathrm{~h}$ and the colonies formed were collected. Plasmid-containing transformants were isolated, reintroduced into $E$. coli $\mathrm{DH} 5 \alpha$ cells, and the transformation mixture was spread onto LB agar plates. The plates were incubated at $37^{\circ} \mathrm{C}$ for $24 \mathrm{~h}$. Plasmids from the transformants were isolated.

\section{Drug susceptibility tests}

The minimum inhibitory concentrations (MICs) of the antimicrobial agents were determined by the broth microdilution technique (9). Using 96-well microtiter plates, two-fold serial dilutions of the antimicrobial agents in $100 \mu \mathrm{L}$ of antibiotic medium 3 with an inoculum of $1 \times 10^{3}$ to $1 \times 10^{4}$ colony forming units (CFUs) per logarithmic-phase cell sample were prepared. The concentration range used was 0.01 to $32 \mu \mathrm{g} / \mathrm{mL}$. MIC is defined as the lowest concentration of the antimicrobial agent that inhibits visible growth after 18 to $24 \mathrm{~h}$ of incubation at $37^{\circ} \mathrm{C}$. The MICs reported here represent the mean values of quadruplicate experiments.

\section{Isolation of ciprofloxacin-resistant mutants}

Spontaneous ciprofloxacin-resistant mutants were selected by plating a $0.1 \mathrm{~mL}$ sample of an overnight culture of the selected strain in Mueller-Hinton broth (final inoculum $10^{7}-10^{8} \mathrm{CFU} / \mathrm{mL}$ ) onto Mueller-Hinton agar plates containing ciprofloxacin at a concentration of approximately $12 \times$ the MIC $(0.8 \mu \mathrm{g} / \mathrm{mL})$ for the selected S. Typhi isolate. After $48 \mathrm{~h}$ of incubation at $37^{\circ} \mathrm{C}$, the grown colonies were counted and streaked onto another plate containing the same concentration of ciprofloxacin to obtain pure colonies of the ciprofloxacin-resistant mutants. The mutation frequency was calculated by dividing the number of resistant cells by the number of viable cells in the original sample.

Table 1. Strains and plasmids used in this study.

\begin{tabular}{|c|c|c|}
\hline Strain or plasmid & Relevant properties & Source or reference \\
\hline \multicolumn{3}{|l|}{ Strains } \\
\hline E. coli DH5 $\alpha$ & supE44 LacUl69 (80 lacM15) hsdR17 recA1 endA1 gyrA96 thi-1relA1 & Biolabs \\
\hline \multicolumn{3}{|c|}{ 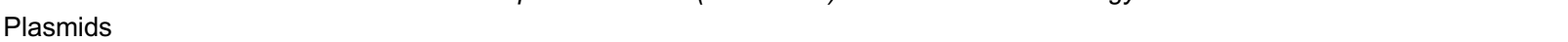 } \\
\hline pUC19 & Multicopy vector, AMPr & Biolabs \\
\hline pGEM-T & PCR cloning vector & Promega \\
\hline \multicolumn{3}{|c|}{ E. coli $\mathrm{DH} 5 \alpha$ clones harboring plasmids } \\
\hline STM1-A & $\mathrm{CPN}^{\mathrm{r}}, \mathrm{OFN}{ }^{\mathrm{r}}, \mathrm{NFN}^{\mathrm{r}}, \mathrm{CFM}^{\mathrm{s}}, \mathrm{CMR}^{\mathrm{s}}, \mathrm{TET}^{\mathrm{s}}, \mathrm{GM}^{\mathrm{s}}$ & This study \\
\hline STM1-B & $\mathrm{CPN}^{\mathrm{s}}, \mathrm{OFN}^{\mathrm{s}}, \mathrm{NFN}^{\mathrm{s}}, \mathrm{CFM}^{\mathrm{s}}, \mathrm{CMR}^{\mathrm{s}}, \mathrm{TET}^{\mathrm{s}}, \mathrm{GM}^{\mathrm{s}}$ & This study \\
\hline STM1-A1 & $\mathrm{CPN}^{\mathrm{r}}, \mathrm{OFN}^{\mathrm{r}}, \mathrm{NFN}^{\mathrm{r}}, \mathrm{CFM}^{\mathrm{s}}, \mathrm{CMR}^{\mathrm{s}}, \mathrm{TET}^{\mathrm{s}}, \mathrm{GM}^{\mathrm{s}}$ & This study \\
\hline STM1-A11 & $\mathrm{CPN}^{r}, \mathrm{OFN}^{r}, \mathrm{NFN}^{r}, \mathrm{CFM}^{\mathrm{s}}, \mathrm{CMR}^{\mathrm{s}}, \mathrm{TET}^{\mathrm{s}}, \mathrm{GM}^{\mathrm{s}}$ & This study \\
\hline
\end{tabular}

STM1: spontaneous fluoroquinolone-resistant mutant; CPN: ciprofloxacin; OFN: ofloxacin; NFN: norfloxacin; CFM: cefotaxime; CMR: chloramphenicol; TET: tetracycline; GM: gentamicin; AMP: ampicillin. 
Protein analysis of crude bacterial extracts

Crude bacterial cell extracts, prepared as described by Sambrook et al. (7), were analyzed by sodium dodecyl sulfate-polyacrylamide gel electrophoresis (SDS-PAGE). A lower separation gel (10\% acrylamide and $0.1 \%$ SDS in $0.375 \mathrm{M}$ Tris- $\mathrm{HCl}$ buffer, $\mathrm{pH} 8.8$ ) and upper stacking gel $(5.0 \%$ acrylamide and $0.1 \%$ SDS in $0.125 \% \mathrm{M}$ Tris- $\mathrm{HCl}$ buffer, pH 6.8) were used.

\section{DNA gyrase assays}

DNA gyrase was isolated and purified according to a method described previously (10). Relaxed Bluescript II plasmid substrate DNA (Agilent technologies, USA) was prepared by treatment of the closed circular plasmid DNA with topoisomerase I according to the manufacturer's recommended protocol.

DNA supercoiling assays were performed as described previously (11). One unit of gyrase is defined as the amount of enzyme required to catalyze the conversion of one-half of a relaxed closed circular DNA to the supercoiled form in $30 \mathrm{~min}$ at $37^{\circ} \mathrm{C}$ in a standard gyrase reaction containing $0.4 \mu \mathrm{g}$ of DNA.

$\mathrm{IC}_{50}$ is defined as the fluoroquinolone concentration that inhibits $50 \%$ of the supercoiling activity of gyrase in a standard gyrase reaction. A control reaction without quinolone was included. After staining the gel in $0.5 \mu \mathrm{g} / \mathrm{mL}$ of ethidium bromide, the $\mathrm{IC}_{50}$ was determined by visual comparison with the control reaction.

\section{Western blot analysis of RecA}

The level of RecA expression in crude bacterial extracts was determined with rabbit antiserum specific for the RecA protein (7). Equal amounts (30 $\mu \mathrm{g}$ each) of each protein sample were separated by SDS-PAGE on a $10 \%$ gel. The gel was then equilibrated in transfer buffer containing $10 \mathrm{mM}$ Tris base, $200 \mathrm{mM}$ glycine, and 10\% methanol for $5 \mathrm{~min}$ before it was electroblotted onto a polyvinylidene difluoride membrane (Applied Biosystems). Immunocomplexed proteins were detected by alkaline phosphatase-conjugated secondary antibodies and the chromatic substrates nitroblue tetrazolium and 5-bromo-4chloro-3-indolylphosphate.

\section{Results}

\section{Isolation of ciprofloxacin-resistant mutants}

Three ciprofloxacin-resistant mutants (STM1-STM3) were isolated from the $S$. Typhi isolate. The mutation frequency of the isolated mutants ranged between $10^{-8}$ and $10^{-7}$. Therefore, the isolated mutants were mostly obtained by single-step mutation.

\section{In-vitro susceptibility testing}

As shown in Table 2, the MICs of the fluoroquinolones tested against the selected mutants increased by $16-63 \times$ those of the parent strain. However, for the other antibiotics tested, in comparison with the parental line, no change in the sensitivity of the mutants was noted.

\section{Inhibition of DNA gyrase supercoiling activity by ciprofloxacin}

The inhibitory effect of ciprofloxacin on DNA gyrase supercoiling activity was measured by a supercoiling assay. The results showed that the $\mathrm{IC}_{50}$ of ciprofloxacin against the DNA gyrase enzymes isolated from two mutants, STM2 and STM3, increased by $5-10 \times$ that of

Table 2. Fluoroquinolone sensitivity of the $S$. Typhi parent strain and its mutants and $E$. coli $\mathrm{DH} 5 \alpha$ and its clones to different antimicrobial agents.

\begin{tabular}{llllllll}
\hline Strains & \multicolumn{7}{c}{ MICs $(\mu \mathrm{g} / \mathrm{mL})$} \\
\cline { 2 - 8 } & CPN & OFN & NFN & CFM & CMR & TET & GM \\
\hline Salmonella Typhi parent & 0.063 & 0.125 & 0.25 & 0.5 & 2.0 & 1.0 & 0.5 \\
STM1 mutant & 2.0 & 4.0 & 4.0 & 0.5 & 2.0 & 1.0 & 0.5 \\
STM2 mutant & 4.0 & 4.0 & 8.0 & 0.5 & 2.0 & 1.0 & 0.5 \\
STM3 mutant & 1.0 & 2.0 & 4.0 & 0.5 & 2.0 & 1.0 & 0.5 \\
E. coli DH5 $\alpha$ & 0.032 & 0.063 & 0.063 & 0.25 & 1.0 & 0.5 & 0.125 \\
E. coli DH5 $\alpha$ containing pUC19 & 0.032 & 0.063 & 0.063 & 0.25 & 1.0 & 0.5 & 0.125 \\
E. coli DH5 $\alpha$ harboring plasmids & & & & & & & \\
STM1-A & 2.0 & 4.0 & 4.0 & 0.25 & 1.0 & 0.5 & 0.125 \\
STM1-B & 0.032 & 0.063 & 0.063 & 0.25 & 1.0 & 0.5 & 0.125 \\
STM1-A1 & 2.0 & 4.0 & 4.0 & 0.25 & 1.0 & 0.5 & 0.125 \\
STM1-A11 & 2.0 & 4.0 & 4.0 & 0.25 & 1.0 & 0.5 & 0.125 \\
\hline
\end{tabular}

Minimum inhibitory concentrations (MIC) are reported as the means of quadruplicate experiments, determined by the broth microdilution technique (9). STM1: spontaneous fluoroquinolone-resistant mutant; CPN: ciprofloxacin; OFN: ofloxacin; NFN: norfloxacin; CFM: cefotaxime; CMR: chloramphenicol; TET: tetracycline; GM: gentamicin. 
the parent strain. However, no change in the ciprofloxacin sensitivity of the DNA gyrase from the STM1 mutant as compared with that of its parent strain was observed. Therefore, DNA gyrase from the STM1 mutant played no role in the STM1 ciprofloxacin resistance phenotype. Accordingly, the STM1 mutant was selected for further examination to clarify the mechanism of resistance to fluoroquinolones.

\section{Cloning S. Typhi to investigate its genetic determinants of fluoroquinolone resistance}

During identification of the genetic determinants of fluoroquinolone resistance in STM1, a shotgun genomic library of this mutant strain was constructed using plasmid pUC19 as the cloning vector. The resulting shotgun library cocktail was introduced into $E$. coli $\mathrm{DH} 5 \alpha$ by transformation, and the transformants were selected on LB agar plates containing $100 \mu \mathrm{g}$ of ampicillin $/ \mathrm{mL}$ and $0.5 \mu \mathrm{g}$ of ciprofloxacin $/ \mathrm{mL}$. One colony (STM1-A) was obtained, and the presence of recombinant plasmids in this clone was confirmed by restriction enzyme digestion. The MICs of ciprofloxacin, ofloxacin, norfloxacin, cefotaxime, chloramphenicol, tetracycline, and gentamicin were determined for this clone. As shown in Table 2, the STM1-A-harboring E. coli clone only exhibited phenotypic resistance to ciprofloxacin, ofloxacin, and norfloxacin. Further studies were carried out to molecularly characterize this clone.

\section{Molecular characterization of the STM1-A clone}

The $7.0 \mathrm{~kb}$ chromosomal insert from STM1-A, digested with EcoRI and Hindlll restriction endonucleases, generated two DNA fragments of approximately 4 and $3 \mathrm{~kb}$ (Figure 1). These two DNA fragments were cloned separately into pUC19, thereby forming two plasmids, STM1-A1 and STM1-A2, for subcloning analysis. The MICs of the $E$. coli clones harboring the resultant plasmids (STM1-A1 and STM1-A2) were determined. The results (Table 2) revealed that plasmid STM1-A1 harbored the gene that confers the selective fluoroquinolone

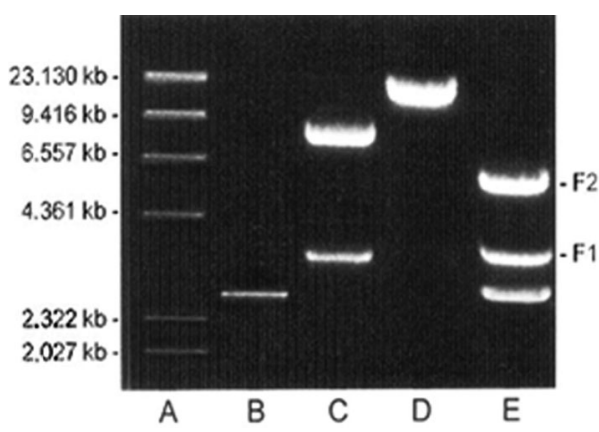

Figure 1. Restriction digest of the 7-kb chromosomal insert from the STM1-A plasmid with EcoRI $(C)$, HindIII $(D)$, and EcoRI and Hind III $(E)$ restriction endonucleases. HindllI digested $\lambda$ DNA $(A)$; pUC19 alone $(B)$. resistance phenotype in $E$. coli $\mathrm{DH} 5 \alpha$. In contrast, the E. coli clone harboring the other plasmid (STM1-A2) had the same degree of fluoroquinolone sensitivity as that of $E$. coli $\mathrm{DH} 5 \alpha$. The nucleotide sequence of the $4 \mathrm{~kb}$ insert from STM1-A1 was determined and subsequently analyzed by a homology search against sequences in the non-redundant database of $S$. Typhi at the $\mathrm{NCBI}$ with the BLAST program. The results of the sequence comparison indicate that the $4 \mathrm{~kb}$ insert of STM1-A1 contains an intact open reading frame that shared identity in the nucleotide sequence of Salmonella enterica subsp. enterica serovar Typhi str. CT18 with the $\operatorname{rec} A$ gene (Accession No. AL513382.1). Thus, the recA gene may play a role in the development of selective resistance against fluoroquinolones.

The $r e c A$ sequence, including the putative ribosomal binding site preceding it, was PCR amplified from STM1-A1. The primers were 5'-CCATGGATGGCTATCGA CGAAAAC-3' (Ncol forward primer) and 5'-TTCGAAT TAAAAATCTTCGTTGG-3' (Hindlll reverse primer). The PCR products were purified, cloned into a pGEM-T plasmid vector, and introduced into $E$. coli $\mathrm{DH} 5 \alpha$. The MICs for the $E$. coli clones harboring the resultant plasmid (STM1-A11) were determined. The results (Table 2) revealed that the $E$. coli clone harboring the STM1-A11 plasmid retained its resistance to fluoroquinolones.

The recA gene was also PCR amplified from the genomic DNA of wild-type S. Typhi and cloned into pGEM-T. Its nucleotide sequence was determined and compared with that of STM1-A11. A 100\% homology score between the two nucleotide sequences was obtained.

The DNA gyrase assay results (Table 3 ) showed the same $I_{50}$ of ciprofloxacin against the DNA gyrase enzymes isolated from $E$. coli $\mathrm{DH} 5 \alpha$ and the $E$. coli clones harboring the STM1-A1, STM1-A2, and STM1-A11 plasmids. Therefore, DNA gyrase was not involved in the development

Table 3. Ciprofloxacin concentrations that inhibited the supercoiling activity of the DNA gyrase enzymes extracted from the S. Typhi parent strain and its mutants, E. coli $\mathrm{DH} 5 \alpha$, and the E. coli clones constructed in this study.

\begin{tabular}{lc}
\hline Source of DNA gyrase & $\mathrm{IC}_{50}(\mu \mathrm{g} / \mathrm{mL})$ \\
\hline S. Typhi parent strain & 0.2 \\
STM1 mutant & 0.2 \\
STM2 mutant & 2.0 \\
STM3 mutant & 1.0 \\
E. coli DH5 $\alpha$ & 0.1 \\
E. coli clones harboring plasmids & \\
STM1-A1 & 0.1 \\
STM1-A2 & 0.1 \\
STM1-A11 & 0.1 \\
\hline
\end{tabular}

$\mathrm{IC}_{50}$ is defined as the fluoroquinolone concentration that inhibits $50 \%$ of the supercoiling activity of gyrase in a standard gyrase reaction. STM1: spontaneous fluoroquinolone-resistant mutant. 
of selective phenotypic fluoroquinolone resistance among the E. coli clones we obtained.

\section{Protein analysis of crude bacterial extracts}

The SDS-PAGE analysis showed there was overexpression of proteins of approximately $40 \mathrm{kDa}$ in crude extracts (Figure 2) from the $E$. coli clones harboring STM1-A1 and STM1-A11 plasmids as compared with crude extracts from E. coli $\mathrm{DH} 5 \alpha$. Additionally, where the E. coli clones harbored pUC19 plasmids alone, no obvious difference in the protein profile was observed when compared with that of $E$. coli $\mathrm{DH} 5 \alpha$.

Western blot analysis using an anti-recA antibody confirmed that the overexpressed $40-\mathrm{kDa}$ protein in the E. coli clones harboring STM1-A1 and STM1-A11 was that of the RecA protein (Figure 3).

\section{Discussion}

RecA, a multifunctional protein, is involved in the induction of SOS responses. In response to an SOSinducing treatment or condition, a signal (involving regions of single-stranded DNA) is generated that stimulates expression of the SOS regulon. RecA protein binding to single-stranded DNA regions in the presence of a nucleoside triphosphate forms a nucleoprotein filament and converts RecA protein to an activated form (12).

Fluoroquinolones induce a certain degree of DNA damage or interference with DNA replication in cells (13). This, in turn, could serve as a signal to activate the coprotease activity of RecA to trigger the SOS regulatory system cascade, resulting in a higher level of resistance to fluoroquinolones. In one study, RecA involvement in fluoroquinolone resistance was inferred by the increase

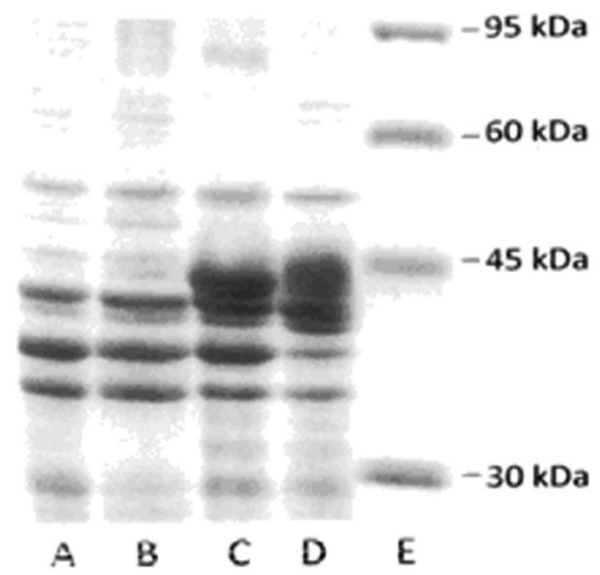

Figure 2. Coomassie brilliant blue stained SDS-PAGE result showing the protein content of crude extracts from samples. Lane A: $E$ coli $\mathrm{DH} 5 \alpha$ host cells grown in Luria-Bertani broth. Lanes $B-D$ : recombinant $E$. coli cells harboring pUC19, STM1-A1, and STM1-A11, respectively. Lane E: protein size standard.

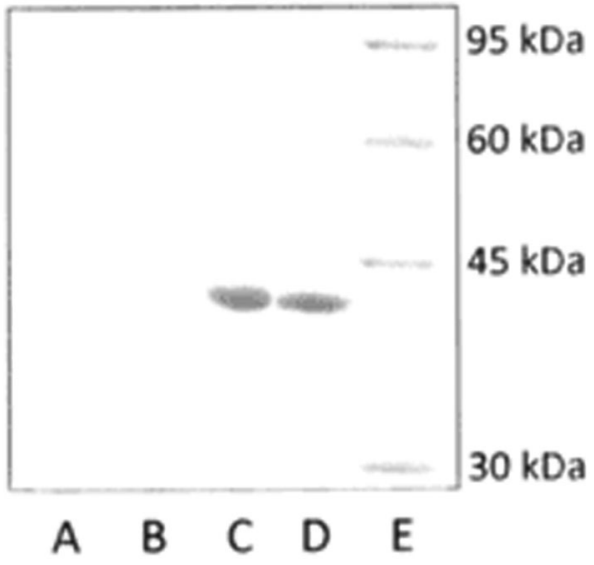

Figure 3. Western blot of RecA protein. Lane A: E coli DH5 $\alpha$ host cells grown in Luria-Bertani broth. Lanes $B-D$ : $E$ coli recombinant cells harboring pUC19, STM1-A1, and STM1-A11, respectively. Lane E: protein size standard.

in the sensitivity of RecA mutants to these compounds (5). RecA expression increases the level of resistance to fluoroquinolones in $E$. coli $\mathrm{DH} 5 \alpha$ through its function in the SOS response. RecA protein plays an important role in the coordinated expression of the SOS regulatory system in response to DNA damage. Additionally, there is an absolute requirement for $\operatorname{rec} A$ in all homologous recombination in $E$. coli where it catalyzes synapsis and strand exchange between homologous molecules $(14,15)$. Piddock and Walters (16) studied the bactericidal effects of various fluoroquinolones on different strains of $E$. coli with mutations in genes for the SOS response. They observed that mutants with constitutive RecA expression survived longer than the wild-type $E$. coli strain.

In the present study, an S. Typhi STM1 mutant was isolated by a single-step mutation. STM1 was characterized by its selective resistance to fluoroquinolones. The results of a DNA gyrase assay revealed that the DNA gyrase isolated from this mutant played no role in the selective fluoroquinolone resistance phenotype. In addition, the E. coli clone that harbored the STM1-A1 plasmid had the same fluoroquinolone resistance phenotype. Sequence analysis indicated that the $4 \mathrm{~kb}$ insert in STM1-A11 contained the recA gene. When the recA gene sequence was PCR amplified from STM1-A11 DNA, and then purified, cloned, and introduced into E. coli $\mathrm{DH} 5 \alpha$, only one of the $E$. coli clones obtained that contained the recA gene exhibited selective fluoroquinolone resistance. Accordingly, RecA expression in the S. Typhi STM1 mutant in E. coli $\mathrm{DH} 5 \alpha$ conferred selective fluoroquinolone resistance. To confirm this finding, the recA gene was PCR amplified from the genomic DNA of wild-type $S$. Typhi and then cloned into pGEM-T. The sequence of the inserted region ( $\operatorname{rec} A$ gene) was determined and compared with that of STM1-A11. The 
results showed complete identity between the two nucleotide sequences.

RecA expression in the $E$. coli clones from this study was investigated by comparative protein analysis of the crude extract and crude outer membrane proteins of the clones, and compared with the protein analysis of the wildtype E. coli $\mathrm{DH} 5 \alpha$. The results of the protein analysis showed there was overexpression of RecA protein in crude extracts and crude outer membrane proteins of the $E$. coli clones harboring STM1-A1 and STM1-A11 plasmids, as compared with the expression profile of $E$. coli $\mathrm{DH} 5 \alpha$. RecA protein expression in E. coli clones harboring STM1-A1 or STM1-A11 in samples of crude outer membrane proteins was confirmed by western blot analysis. The increased association of the RecA protein with the membrane fractions that was observed in this study requires the presence of the activated form of RecA and this association may contribute substantively to the SOS response $(17,18)$. Therefore, overexpression of RecA confers resistance to fluoroquinolones in $E$. coli $\mathrm{DH} 5 \alpha$.

Accordingly, the results suggest that transformation of the recA gene of $S$. Typhi into E. coli $\mathrm{DH} 5 \alpha$ resulted in RecA protein expression and development of the selective fluoroquinolone resistance phenotype in the E. coli $\mathrm{DH} 5 \alpha$ clone.

\section{Acknowledgments}

This research was supported by the Deanship of Scientific Research (DSR), King Abdulaziz University, Jeddah, Saudi Arabia (\#166-609-D1435). The authors wish to thank the technical and financial support from the DSR.

\section{References}

1. Kumar A, Pandit V, Shetty S, Rao CR, Pattanshetty S, Samarasinghe CM. Study of clinical profile and antibiotic sensitivity pattern in culture-positive typhoid fever cases. Indian J Community Med 2012; 37: 256-258.

2. Koirala KD, Thanh DP, Thapa SD, Arjyal A, Karkey A, Dongol S, et al. Highly resistant Salmonella enterica serovar Typhi with a novel gyrA mutation raises questions about the long-term efficacy of older fluoroquinolones for treating typhoid fever. Antimicrob Agents Chemother 2012; 56: 2761-2762, doi: 10.1128/AAC.06414-11.

3. Tenover FC. Mechanisms of antimicrobial resistance in bacteria. Am J Infect Control 2006; 34: S3-10.

4. Piddock LJV, Wise R. Induction of the SOS responsein Escerichia coli by 4-quinolone antimicrobial agents. FEMS Microbiol 1987; 41: 289-294, doi: 10.1111/j.1574-6968.1987. tb02213.x.

5. Singh R, Ledesma KR, Chang KT, Tam VH. Impact of recA on levofloxacin exposure-related resistance development. Antimicrob Agents Chemother 2010; 54: 4262-4268, doi: 10.1128/AAC.00168-10.

6. Aranda J, Bardina C, Beceiro A, Rumbo S, Cabral MP, Barbe $\mathrm{J}$, et al. Acinetobacter baumannii RecA protein in repair of DNA damage, antimicrobial resistance, general stress response, and virulence. J Bacteriol 2011; 193: 3740-3747, doi: 10.1128/JB.00389-11.

7. Sambrook J, Fritsch EF, Maniatis T. Molecular cloning, a laboratory manual. New York: Cold Spring Harbor; 1989.

8. Sanger $F$, Nicklen $S$, Coulson AR. DNA sequencing with chain-terminating inhibitors. Proc Natl Acad Sci U S A 1977; 74: 5463-5467, doi: 10.1073/pnas.74.12.5463.

9. Clinical and Laboratory Standards Institute (CLSI). Performance standards for antimicrobial susceptibility testing. In: Anonymous (Editor), 19th Informational Supplement Document, Vol. 29; 15-30. Wayne, PA. 2009.

10. Gellert M, Fisher LM, O'Dea MH. DNA gyrase: purification and catalytic properties of a fragment of gyrase $B$ protein. Proc Natl Acad Sci U S A 1979; 76: 6289-6293, doi: 10.1073/pnas.76.12.6289.
11. Mizuuchi K, Mizuuchi M, O’Dea MH, Gellert M. Cloning and simplified purification of Escherichia coli DNA gyrase A and B proteins. J Biol Chem 1984; 259: 9199-9201.

12. Adikesavan AK, Katsonis P, Marciano DC, Lua R, Herman $C$, Lichtarge O. Separation of recombination and SOS response in Escherichia coli RecA suggests LexA interaction sites. PLoS Genet 2011; 7: e1002244, doi: 10.1371/ journal.pgen.1002244.

13. MacGuire AE, Ching MC, Diamond BH, Kazakov A, Novichkov P, Godoy VG. Activation of phenotypic subpopulations in response to ciprofloxacin treatment in Acinetobacter baumannii. Mol Microbiol 2014; 92: 138-152, doi: 10.1111/mmi.12541.

14. Frischkorn K, Sander P, Scholz M, Teschner K, Prammananan T, Bottger EC. Investigation of mycobacterial recA function: protein introns in the RecA of pathogenic mycobacteria do not affect competency for homologous recombination. Mol Microbiol 1998; 29: 1203-1214, doi: 10.1046/j.1365-2958.1998. 01003.x.

15. Papavinasasundaram KG, Colston MJ, Davis EO. Construction and complementation of a recA deletion mutant of Mycobacterium smegmatis reveals that the intein in Mycobacterium tuberculosis recA does not affect RecA function. Mol Microbiol 1998; 30: 525-534, doi: 10.1046/j.13652958.1998.01083.x.

16. Piddock LJ, Walters RN. Bactericidal activities of five quinolones for Escherichia coli strains with mutations in genes encoding the SOS response or cell division. Antimicrob Agents Chemother 1992; 36: 819-825, doi: 10.1128/AAC.36.4.819.

17. Garvey N, St John AC, Witkin EM. Evidence for RecA protein association with the cell membrane and for changes in the levels of major outer membrane proteins in SOS-induced Escherichia coli cells. J Bacteriol 1985; 163: 870-876.

18. Schroder W, Goerke C, Wolz C. Opposing effects of aminocoumarins and fluoroquinolones on the SOS response and adaptability in Staphylococcus aureus. J Antimicrob Chemother 2013; 68: 529-538, doi: 10.1093/jac/dks456. 